\title{
Interaction of a graphene sheet with a ferromagnetic metal plate
}

\author{
Anh D. Phan,,${ }^{1,}$ N. A. Viet, ${ }^{2}$ Nikolai A. Poklonski, ${ }^{3}$ Lilia M. Woods, ${ }^{1}$ and Chi H. Le ${ }^{4}$ \\ ${ }^{1}$ Department of Physics, University of South Florida, Tampa, Florida 33620, USA \\ ${ }^{2}$ Institute of Physics, 10 Daotan, Badinh, Hanoi, Vietnam \\ ${ }^{3}$ Physics Department, Belarusian State University, Minsk 220030, Belarus \\ ${ }^{4}$ School of Engineering, University of Greenwich, Medway, United Kingdom
}

(Received 24 July 2012; published 12 October 2012)

\begin{abstract}
Dispersion forces, such as Casimir and van der Waals forces, can have a significant influence on the fabrication, handling, and assembly processes as well as the performance of micro- and nanodevices. In this paper, we investigate the Casimir force between a graphene sheet and a ferromagnetic metallic substrate in vacuum. The reflection coefficients, entering the expression for the force, are dependent on the graphene conductivity, which is described by the Kubo formalism. It is found that the magnetic response of the ferromagnet plays a significant role at low temperatures or high value of the graphene chemical potential. The numerical results also demonstrate that the thickness of the metallic plate has a minor influence on the Casimir force. This study maybe useful for the design and operation of micro- and nanodevices.
\end{abstract}

DOI: $10.1103 /$ PhysRevB.86.155419

PACS number(s): 78.20.Ls, 31.30.jh, 78.67.Wj, 12.20.Fv

\section{INTRODUCTION}

Micro- and nanotechnologies are considered as enabling technologies with exceptional economic capabilities. There are increasingly more products in today's market in which the integrated micro- and nanoelectromechanical systems (MEMS/NEMS) are useful, because they allow for improved functionality, lower costs, and higher quality. With the decrease in the size of MEMS and NEMS, additional nanoscale surface forces, such as the Casimir and van der Waals forces, should be considered, ${ }^{1,2}$ especially in the areas of micro and nano machining, fabrication, manipulation, assembly, and metrology. Therefore, a fundamental understanding of the Casimir force has recently been under intense discussion.

The Casimir force can cause small elements in a device to stick together. Also, such nanoscale surface forces may overcome elastic restoring actions in the device and lead to sticktion effects during the fabrication process. ${ }^{1}$ It has been recently shown, that Casimir forces may hamper the functioning of MEMS and NEMS devices by providing a pull-in instability. ${ }^{3}$ The Casimir force between two objects can induce further adhesion and stiction, leading to failures in devices. ${ }^{2-5}$ Particularly, when the size of a material is under the threshold length, the influence of the loading interaction on the design and manufacture of micro- and nanodevices becomes much more prominent.

The Casimir interaction originates from the quantum electromagnetic fluctuations between two objects. ${ }^{2,5,6}$ It provides a fundamental understanding about nanoscience and can play a significant role in the performance of devices. For over six decades, the Lifshitz theory has been employed to investigate this force in metal-metal, ${ }^{7,8}$ semiconductor-semiconductor, ${ }^{9}$ metal-superconductor, ${ }^{10,11}$ metamaterials-metamaterials, ${ }^{12,13}$ and graphene-graphene systems. ${ }^{14-17}$ The results of some configurations have been experimentally examined and have good agreement with theoretical calculations. ${ }^{5,18}$

The interaction between a nonmagnetic metal with a magnetodielectric material has also recently been discussed. ${ }^{19-21}$ These studies illustrate that the magnetic properties can significantly influence the Casimir pressure. Theoretical and experimental studies also show that it is possible to obtain a repulsive Casimir force in such systems, ${ }^{2,20,22}$ and the results could provide possible solutions to handle the stiction and adhesion problems found in MEMS/NEMS devices. In terms of theory, the calculations depend substantially on the model that describes the dielectric function of metals.

Graphene, an atomically thin layered material with novel properties, has gained a great deal of attention since its discovery. ${ }^{23}$ Researchers from a wide range of scientific fields have spent considerable effort in exploring its nature and its potential for practical applications. ${ }^{24-27}$ Recently obtained results demonstrate that graphene can be a promising candidate for the next-generation electronic devices. ${ }^{28}$

In this paper, we calculate the Casimir force between a graphene layer and a ferromagnetic (FM) substrate, as shown in Fig. 1. The FM material is taken to be Fe described via dielectric and magnetic response properties. We utilize the Lifshitz theory to examine the role of the separation, temperature, and thickness of the substrate. We also investigate the influence of the magnetic response of the FM and show that in most cases its contribution is relatively small compared to the one from the dielectric response.

This paper is organized as follows: Sec. II presents detailed calculations of the thermal Casimir interactions. The results and discussion are given in Sec. III. Finally, Sec. IV presents the conclusions.

\section{CASIMIR INTERACTION BETWEEN GRAPHENE AND A METAL SUBSTRATE}

Here we calculate the Casimir force between a graphene sheet and a FM substrate in vacuum at a separation $a$. The force per unit area at temperature $T$ is given as ${ }^{5,15,29,30}$

$$
\begin{aligned}
F(a, T)= & -\frac{k_{B} T}{\pi} \sum_{l=0}^{\infty}\left(1-\frac{1}{2} \delta_{l 0}\right) \int_{0}^{\infty} q_{l} k_{\perp} d k_{\perp} \\
& \times\left(\frac{r_{T E}^{(1)} r_{T E}^{(2)}}{e^{2 q_{l} a}-r_{T E}^{(1)} r_{T E}^{(2)}}+\frac{r_{T M}^{(1)} r_{T M}^{(2)}}{e^{2 q_{l} a}-r_{T M}^{(1)} r_{T M}^{(2)}}\right),
\end{aligned}
$$




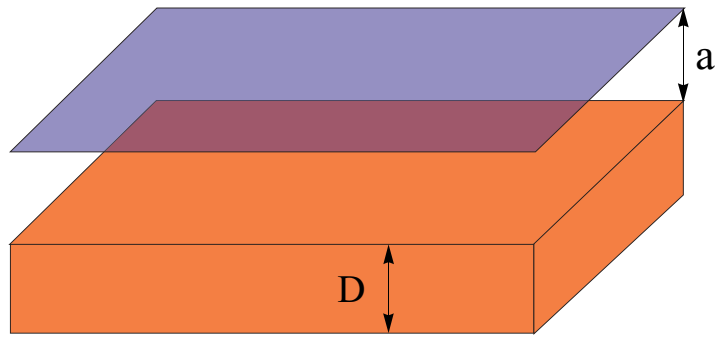

FIG. 1. (Color online) Schematics of a two-dimensional layer (graphene) and a substrate (FM metal) with thickness $D$.

where $k_{B}$ is the Boltzmann constant, and $r_{T M}^{(1,2)}$ and $r_{T E}^{(1,2)}$ are the reflection coefficients corresponding, respectively, to the transverse magnetic (TM) and transverse electric (TE) field modes.

The reflection coefficients for the substrate are given as $^{2,19,20}$

$$
\begin{aligned}
& r_{T E}^{(1)} \equiv r_{T E}^{(1)}\left(i \xi_{l}, k_{\perp}\right)=\frac{\mu_{1}\left(i \xi_{l}\right) q_{l}-k_{1}}{\mu_{1}\left(i \xi_{l}\right) q_{l}+k_{1}} \\
& r_{T M}^{(1)} \equiv r_{T M}^{(1)}\left(i \xi_{l}, k_{\perp}\right)=\frac{\varepsilon_{1}\left(i \xi_{l}\right) q_{l}-k_{1}}{\varepsilon_{1}\left(i \xi_{l}\right) q_{l}+k_{1}}
\end{aligned}
$$

where

$$
\begin{aligned}
& q_{l} \equiv q_{l}\left(i \xi_{l}, k_{\perp}\right)=\sqrt{k_{\perp}^{2}+\frac{\xi_{l}^{2}}{c^{2}}}, \\
& k_{1} \equiv k_{1}\left(i \xi_{l}, k_{\perp}\right)=\sqrt{k_{\perp}^{2}+\mu_{1}\left(i \xi_{l}\right) \varepsilon_{1}\left(i \xi_{l}\right) \frac{\xi_{l}^{2}}{c^{2}}} .
\end{aligned}
$$

Note that $k_{\perp}$ is the wave vector component perpendicular to the plate, $c$ is the speed of light, and $\xi_{l}=2 \pi k_{B} T l / \hbar$ are the Matsubara frequencies. The response properties of the FM metal are characterized by the dielectric function $\varepsilon_{1}\left(i \xi_{l}\right)$ and the permeability function $\mu_{1}\left(i \xi_{l}\right)$, which are frequency dependent along the imaginary axis $\left(\omega_{l}=i \xi_{l}\right)$. We use the Drude model $\varepsilon_{1 D}$ and the plasma model $\varepsilon_{1 P}$ to describe the dielectric function, $5,19,21$

$$
\begin{aligned}
& \varepsilon_{1 D}(i \xi)=1+\frac{\omega_{p}^{2}}{\xi\left(\xi+\gamma_{p}\right)}, \\
& \varepsilon_{1 P}(i \xi)=1+\frac{\omega_{p}^{2}}{\xi^{2}},
\end{aligned}
$$

where $\omega_{p}$ is the plasma frequency and the damping parameter is $\gamma_{p}$. At room temperature, the $l=0$ term of the CasimirLifshitz force in the metallic system is dominant. ${ }^{22,33}$ This allows us to consider the contribution of the static magnetic permeability $\mu(0) \gg 1$. For higher orders of $l$, one should use $\mu\left(\xi_{l}\right)=1 .^{21}$ Both models lead to $r_{T M}^{(1)}(0)=1$. However, we can obtain two different expressions for $r_{T E}^{(1)}(0)$ when applying these two models, ${ }^{21}$

$$
\begin{aligned}
& r_{T E, D}^{(1)}\left(0, k_{\perp}\right)=\frac{\mu(0)-1}{\mu(0)+1}, \\
& r_{T E, P}^{(1)}\left(0, k_{\perp}\right)=\frac{\mu(0) c k_{\perp}-\sqrt{c^{2} k_{\perp}^{2}+\mu(0) \omega_{p}^{2}}}{\mu(0) c k_{\perp}+\sqrt{c^{2} k_{\perp}^{2}+\mu(0) \omega_{p}^{2}}} .
\end{aligned}
$$

Here, $r_{T E, D}^{(1)}\left(0, k_{\perp}\right)$ and $r_{T E, P}^{(1)}\left(0, k_{\perp}\right)$ are the TE reflection coefficients corresponding to the Drude and plasma models, respectively.

For graphene, the reflection coefficients are found to be $\mathrm{e}^{16,22}$

$$
\begin{aligned}
& r_{T E}^{(2)} \equiv r_{T E}^{(2)}\left(i \xi_{l}, k_{\perp}\right)=-\frac{2 \pi \xi_{l} \sigma / q_{l} c^{2}}{1+2 \pi \xi_{l} \sigma / q_{l} c^{2}}, \\
& r_{T M}^{(2)} \equiv r_{T M}^{(2)}\left(i \xi_{l}, k_{\perp}\right)=\frac{2 \pi \sigma q_{l} / \xi_{l}}{1+2 \pi \sigma q_{l} / \xi_{l}},
\end{aligned}
$$

where $\sigma \equiv \sigma(i \xi)$ is the two-dimensional (2D) conductivity described via the the Kubo formalism, ${ }^{16}$

$$
\sigma(i \xi)=\frac{2 e^{2} k_{B} T \ln (2)}{\pi \hbar^{2} \xi}+\frac{e^{2} \xi}{8 \pi k_{B} T} \int_{0}^{\infty} \frac{\tanh (x) d x}{x^{2}+\left(\frac{\hbar \xi}{4 k_{B} T}\right)^{2}} .
$$

The first term in Eq. (7) corresponds to the intraband transitions, while the second term corresponds to the interband transitions. At low temperatures, the graphene conductivity $\sigma\left(i \xi_{l}\right)$ approaches the universal value $\sigma_{0}=e^{2} / 4 \hbar$.

Using Eqs. (6) and (7), the TM reflection coefficient of graphene is given by

$$
r_{T M}^{(2)}=\frac{\frac{2 e^{2} k_{B} T}{\pi \hbar^{2}}+\frac{e^{2} \xi_{l}^{3}}{8 \pi k_{B} T} \int_{0}^{\infty} \frac{\tanh x d x}{x^{2}+\left(\hbar \xi_{l} / 4 k_{B} T\right)^{2}}}{\frac{\xi_{l}^{2}}{2 \pi q_{l}}+\frac{2 e^{2} k_{B} T}{\pi \hbar^{2}}+\frac{e^{2} \xi_{l}^{3}}{8 \pi k_{B} T} \int_{0}^{\infty} \frac{\tanh x d x}{x^{2}+\left(\hbar \xi_{l} / 4 k_{B} T\right)^{2}}} .
$$

Equation (8) suggests $r_{T M}^{(2)}\left(0, k_{\perp}\right)=1$, while

$$
r_{T E}^{(2)}\left(0, k_{\perp}\right)=-\frac{(2 \pi / c) 8 \ln (2) \sigma_{0}}{\pi \lambda_{T} k_{\perp}+(2 \pi / c) 8 \ln (2) \sigma_{0}},
$$

where $\lambda_{T}=\hbar c / k_{B} T$ is the standard thermal wavelength. The explicit expression for $r_{T E}^{(2)}\left(0, k_{\perp}\right)$ shows that it depends on the temperature, and only the intraband transitions from the graphene conductivity make a contribution.

The first Matsubara frequencies $\xi_{1}$ are approximately 0.027 , 0.162 , and $0.325 \mathrm{eV}$, corresponding to the temperature 50, 300, and $600 \mathrm{~K}$, respectively. As seen in Fig. 2, for $l \geqslant 2$, it is possible to substitute $\sigma_{0}$ for $\sigma\left(i \xi_{l}\right)$ in Eq. (6) to calculate the reflection coefficients and the higher-order terms in the Casimir-Lifshitz force formula.

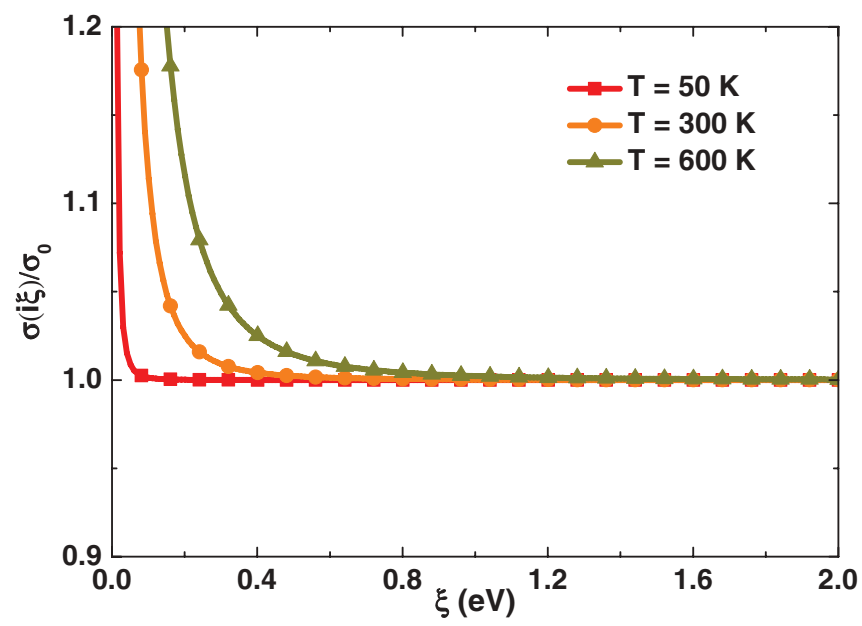

FIG. 2. (Color online) The normalized graphene conductivity $\sigma(i \xi) / \sigma_{0}$ vs frequency at given temperatures. 


\section{RESULTS AND DISCUSSION}

In this section, we consider the Casimir interactions between graphene and a $\mathrm{Fe}$ metal plate. The parameters of $\mathrm{Fe}$ are $\omega_{p}=4.09 \mathrm{eV}, \gamma_{p}=0.018 \mathrm{eV}$, and $\mu(0)=10^{4} .7$ As discussed above for $r_{T M}^{(1)}\left(0, k_{\perp}\right)=r_{T M}^{(2)}\left(0, k_{\perp}\right)=1$, the TM contribution to the $l=0$ term of the Casimir force is given by

$$
F_{T M}^{(0)}(a, T)=-\frac{k_{B} T}{2 \pi} \int_{0}^{\infty} \frac{k_{\perp}^{2} d k_{\perp}}{e^{2 k_{\perp} a}-1}=-\frac{k_{B} T \zeta(3)}{8 \pi a^{3}} .
$$

This expression is identical to the term with $l=0$ in the Casimir force between two metals. The sum of the $l \geqslant 1$ is much smaller than that of the metallic system due to the presence of the low graphene conductivity. ${ }^{16}$ As a result, the $l=0$ term is the dominating term. Now, to calculate the contribution of the TE mode $F_{T E}^{(0)}(a, T)$ with $l=0$, it is necessary to choose a good model for Fe. Both the plasma ${ }^{19}$ and Drude models, ${ }^{31}$ however, have been widely used to compute the Casimir interaction and fit with experimental data. The determination of the accuracy of the two models as compared to experimental data has been a controversial issue. In the following work, we utilize the plasma model to calculate the interactions. Thus $F_{T E}^{(0)}(a, T)$ is expressed by

$$
F_{T E}^{(0)}(a, T)=-\frac{k_{B} T}{2 \pi} \int_{0}^{\infty} \frac{k_{\perp}^{2} d k_{\perp}}{\frac{e^{2 k_{\perp} a}}{r_{T E}^{(1)}\left(0, k_{\perp}\right) r_{T E}^{(2)}\left(0, k_{\perp}\right)}-1} .
$$

Note that $F_{T E}^{(0)}(a, T)$ is a unique term affected by the magnetic property of the ferromagnetic substrate. In Fig. 3, the ratio $F_{T E}^{(0)} / F_{T M}^{(0)}$ is less than $0.75 \%$ for distances $a$ in the $\mu \mathrm{m}$ range at given temperatures. This indicates that the effect of $\mu(0)$ on the Casimir force is small. For this reason, Fe can be treated as a regular nonmagnetic metal $\mu(i \xi)=1$.

For a finite-thickness iron slab $D$, the reflection coefficients of the metal material are modified as follows: ${ }^{20,32}$

$$
R_{T E, T M}\left(i \xi_{l}, k_{\perp}\right)=r_{T E, T M}^{(1)} \frac{1-e^{-2 k_{1} D}}{1-\left(r_{T E, T M}^{(1)}\right)^{2} e^{-2 k_{1} D}} .
$$

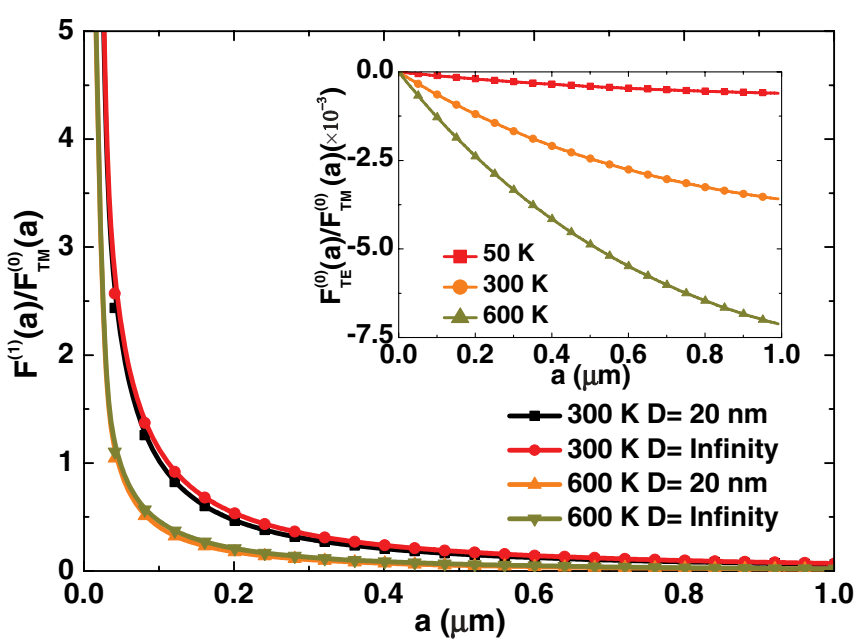

FIG. 3. (Color online) The ratios of $F_{T E}^{(0)}(a, T) / F_{T M}^{(0)}(a, T)$ and $F^{(1)}(a, T) / F_{T M}^{(0)}(a, T)$ at 50,300 , and $600 \mathrm{~K}$ as a function of separation distance $a$.
To study the influence of thickness on the Casimir force, we rewrite Eq. (1) in the following way:

$$
F(a, T)=F_{T M}^{(0)}(a, T)+F_{T E}^{(0)}(a, T)+F^{(1)}(a, T) .
$$

Here, $F^{(1)}(a, T)$ is the sum of all $l \geqslant 1$ terms and is thickness dependent. Because $r_{T M}^{(1)}\left(0, k_{\perp}\right)=1$, therefore $R_{T M}^{(1)}\left(0, k_{\perp}\right)=1$. $F_{T M}^{(0)}(a, T)$ is given in Eq. (10) and it is independent of $D$. The insert in Fig. 3 shows that $F_{T E}^{(0)}(a, T)$ is much weaker than that for the thick plate and one can consider $F_{T E}^{(0)}(a, T) \approx 0$. Therefore, only $F^{(1)}(a, T)$ depends on the substrate thickness.

Figure 3 also shows that even $F^{(1)}(a, T)$ is weakly dependent on $D$ for practically all ranges since reduction of the thickness gives rise to a small decrease of the Casimir force. Because the thermal wavelength of graphene is $\lambda_{T} / 200 \approx 38 \mathrm{~nm}$ at room temperature, at distances beyond the thermal wavelength of a system, the contribution of the sum of $l \geqslant 1$ terms can be ignored. Thus the influence of $F^{(1)}(a, T)$ is not significant. This suggests that the graphene significantly interacts only with the nearest surface layers of the bottom substrate. We find that the universal graphene conductivity is the main reason for less than $2 \%$ difference as one compares the dispersion force between two graphene sheets with $F_{0}(a) .{ }^{16}$ This demonstrates that the approximate formula for the Casimir interaction equivalent to the $l=0$ term of the Casimir-Lifshitz expression in this regime is $F_{\text {app }}(a, T)=-k_{B} T \zeta(3) / 8 \pi a^{3}$. In Fig. 4, we plot the normalized Casimir force with the full expression [see Eq. (1)] and the approximate expression. The $l=0$ term is dominant and can replace the full expression of the Casimir interaction when $a \geqslant 0.8 \mu \mathrm{m}$ for $300 \mathrm{~K}$.

The critical $T_{c}$ of Fe is $1043 \mathrm{~K}$. At $T>T_{c}$, the magnetic properties of the ferromagnetic material nearly vanish. Spins in the Fe slab are rearranged so that $\mu(i \xi)=1$ at all frequencies. Nevertheless, the dielectric function is not affected by the directions of spins. Therefore, the plasma frequency and damping parameter are unchanged by temperature increases at $T \geqslant T_{c}$. If we consider the Casimir interaction between $\mathrm{Fe}$ and another metal, the phase transition leads to a significant change

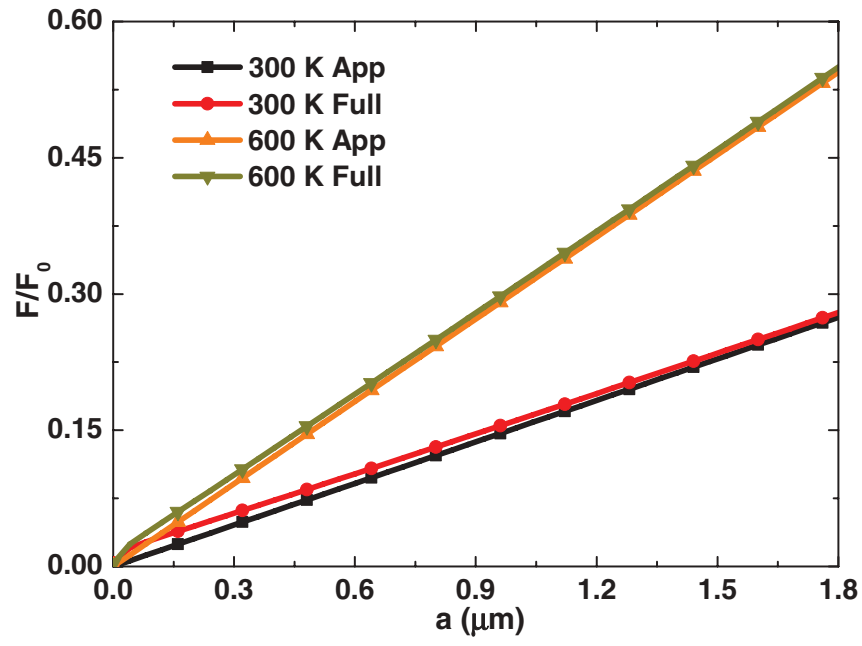

FIG. 4. (Color online) The Casimir pressures corresponding to the approximate and full expression at different temperatures. Here, $F_{0}(a)=-\pi^{2} \hbar c / 240 a^{4}$. 
in the mutual interaction. ${ }^{19,21}$ Due to the fact that graphene is quite transparent with the presence of the magnetic properties, the dispersion force is not affected significantly.

One notes that Eq. (7) is applicable, for undoped graphene with chemical potential $\mu_{C}=0$. If $\mu_{C} \neq 0$, then the graphene conductivity becomes ${ }^{33}$

$$
\begin{aligned}
\sigma\left(\mu_{C}, i \xi\right)= & \frac{e^{2} k_{B} T \ln (2)}{\pi \hbar^{2} \xi}+\frac{e^{2} k_{B} T \ln \left[1+\cosh \left(\mu_{C} / k_{B} T\right)\right]}{\pi \hbar^{2} \xi} \\
& +\frac{e^{2} \xi}{\pi} \int_{0}^{\infty} \frac{\sinh \left(E / k_{B} T\right)}{\cosh \left(E / k_{B} T\right)+\cosh \left(\mu_{C} / k_{B} T\right)} \\
& \times \frac{d E}{(\hbar \xi)^{2}+4 E^{2}} .
\end{aligned}
$$

The first two terms correspond to the intraband transitions; the last term is the interband component. Equation (14) shows that the intraband contribution to $\sigma\left(\mu_{C}, i \xi\right)$ still induces a nonzero value of $r_{T E}^{(2)}\left(0, k_{\perp}\right)$. One way to control the graphene chemical potential is by an applied electric field $E_{d},{ }^{34,35}$

$$
\frac{\pi \varepsilon_{0} \hbar^{2} v_{F}^{2}}{e} E_{d}=\int_{0}^{\infty} E\left[f(E)-f\left(E+2 \mu_{C}\right)\right] d E,
$$

where $f(E)$ is the Fermi distribution function and $v_{F}=c / 300$ is the graphene Fermi velocity. The chemical potential can also be influenced by doping.

In the limit of small chemical potential, $r_{T E}^{(2)}\left(0, k_{\perp}\right)$ is represented as Eq. (9). For $\mu_{C} \gg k_{B} T$, the expression of the TE reflection coefficient with $l=0$ is written as

$$
r_{T E}^{(2)}\left(0, k_{\perp}\right) \approx-\frac{4 \sigma_{0} \mu_{C} /(\pi \hbar c)}{k_{\perp}+4 \sigma_{0} \mu_{C} /(\pi \hbar c)} .
$$

In Fig. 5, the Casimir interaction is calculated at low temperatures $(\leqslant 50 \mathrm{~K})$ and $\mu_{C}=1 \mathrm{eV}$, with and without the magnetic properties. Unlike the case of a pristine graphene sheet $\left(\mu_{C}=0 \mathrm{eV}\right)$, the influence of permeability of Fe on the dispersion force is more pronounced at $10 \mathrm{~K}$. The magnetic properties, however, have much smaller effect on the Casimir force at temperatures greater than $50 \mathrm{~K} . F(a, T)$ is mainly determined by the interband conductivity of graphene. At $10 \mathrm{~K}$, the contribution of the intraband on the Casimir

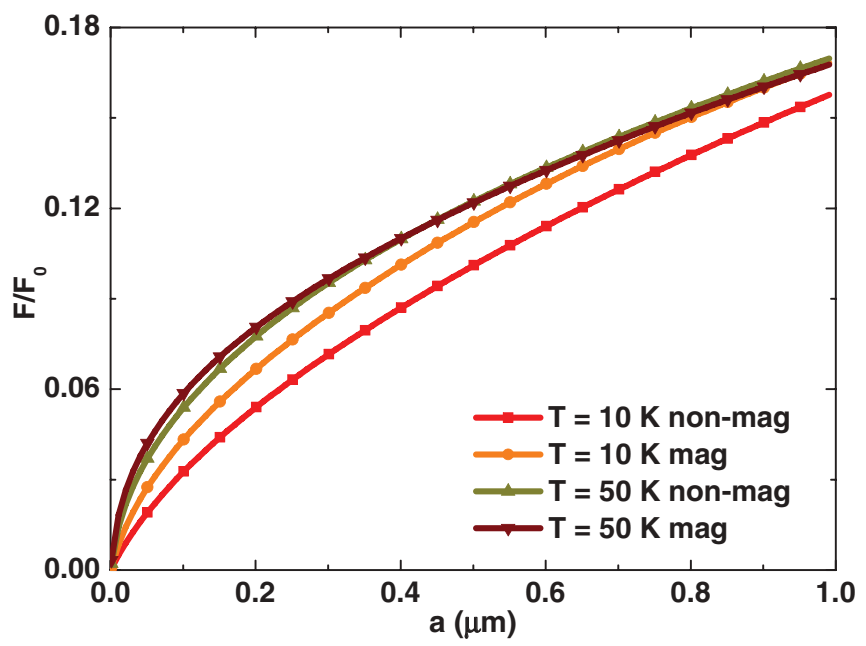

FIG. 5. (Color online) The Casimir force normalized to $F_{0}$ for $\mu=1$ (non-mag) and $\mu_{P}$ (mag) when $\mu_{C}=1 \mathrm{eV}$.

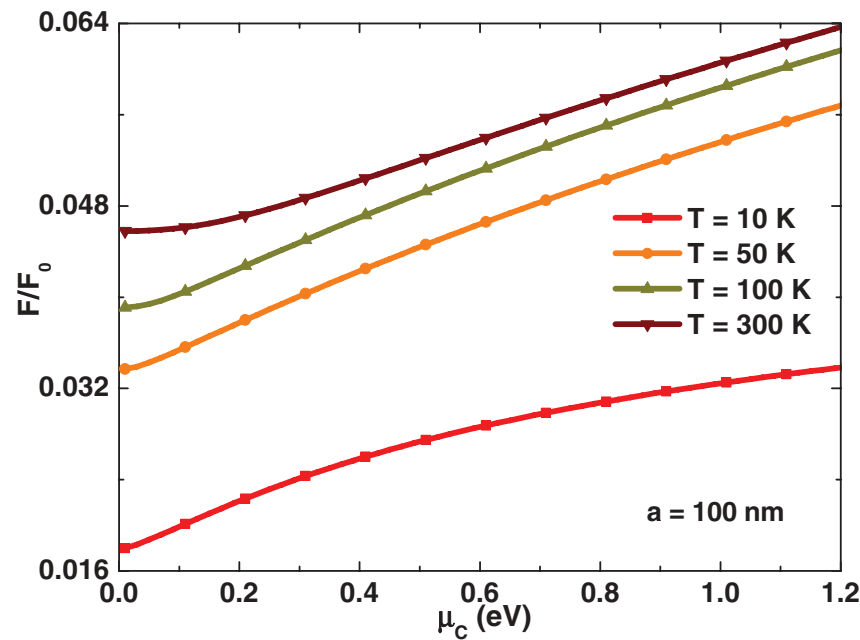

FIG. 6. (Color online) The Casimir force normalized to $F_{0}$ as a function of chemical potential.

force can be considerable compared to that of the interband conductivity. However, as the temperature increases, the interband contribution increases substantially, and causes a decrease in the contribution from intraband. For this reason, the magnetic properties nearly disappear at $T \geqslant 50 \mathrm{~K}$.

To further investigate the Casimir force dependence on chemical potential, we consider the fluctuation interactions at $a=100 \mathrm{~nm}$ and various temperatures versus $\mu_{C}$, as shown in Fig. 6. $F(a, T) / F_{0}$ changes from 0.018 to 0.034 at $T=10 \mathrm{~K}$, and from 0.045 to 0.064 at $T=300 \mathrm{~K}$, when $\mu_{C}$ varies in the regime below $1.2 \mathrm{eV}$. In addition, at higher temperatures, the curves of $F(a, T) / F_{0}$ are of the linear form.

\section{CONCLUSIONS}

When designing and manufacturing micro- and nanodevices and components, it is important to understand the loading and the effects of related forces on the systems and their components, especially due surface forces such as the Casimir and van der Waals. In this study, a comprehensive investigation and discussion of the Casimir interaction between graphene and FM materials is presented. We discuss the Casimir force, which is a function of the graphene conductivity and the permeability constant of Fe. It is shown that the conductivity of graphene heavily depends on the chemical potential, which causes a difference between nonmagnetic and magnetic calculations at the low temperatures. For higher temperatures, the influence of the magnetic properties is small. One can apply a bias electric field to a graphene sheet to tailor the chemical potential of graphene in order to control the magnitude of the Casimir force. The numerical calculations demonstrate that the thickness of a metal slab had a minor influence on the Casimir force. The results from this study maybe useful for the design and operation of micro- and nanodevices utilizing magnetic materials at various temperature regimes.

\section{ACKNOWLEDGMENTS}

We give thanks to D. Drosdoff for discussions. This research was supported by the Nafosted Grant No. 103.06-2011.51. 
L.M.W. acknowledges the US Department of Energy under Contract No. DE-FG02-06ER46297. N.A.P. acknowledges the financial support from the Belarusian Republican Foundation for Fundamental Research Grant No. F11V-001.
*Corresponding author: anhphan@mail.usf.edu

${ }^{1}$ R. C. Batra, M. Porfiri, and D. Spinello, Int. J. Solids Struct. 45, 3558 (2008).

${ }^{2}$ Anh D. Phan and N. A. Viet, Phys. Rev. A 84, 062503 (2011).

${ }^{3}$ R. Ardito, A. Frangi, A. Corigliano, B. De Masi, and G. Cazzaniga, Microelectron. Reliab. 52, 271 (2012).

${ }^{4}$ Alejandro W. Rodriguez, David Woolf, Pui-Chuen Hui, Eiji Iwase, Alexander P. McCauley, Federico Capasso, Marko Loncar, and Steven G. Johnson, Appl. Phys. Lett. 98, 194105 (2011).

${ }^{5}$ G. L. Klimchitskaya, U. Mohideen, and V. M. Mostepanenko, Rev. Mod. Phys. 81, 1827 (2009).

${ }^{6}$ Anh D. Phan and N. A. Viet, Phys. Status Solidi RRL 6, 274 (2012).

${ }^{7}$ M. Bostrom and Bo E. Sernelius, Phys. Rev. Lett. 84, 4757 (2000).

${ }^{8}$ M. Bordag, B. Geyer, G. L. Klimchitskaya, and V. M. Mostepanenko, Phys. Rev. Lett. 85, 503 (2000).

${ }^{9}$ W. J. Kim, A. O. Sushkov, D. A. R. Dalvit, and S. K. Lamoreaux, Phys. Rev. Lett. 103, 060401 (2009).

${ }^{10}$ Giuseppe Bimonte, Enrico Calloni, Giampiero Esposito, Leopoldo Milano, and Luigi Rosa, Phys. Rev. Lett. 94, 180402 (2005).

${ }^{11}$ Giuseppe Bimonte, Phys. Rev. A 78, 062101 (2008).

${ }^{12}$ F. S. S. Rosa, D. A. R. Dalvit, and P. W. Milonni, Phys. Rev. A 78, 032117 (2008).

${ }^{13}$ R. Zhaom, T. Koschny, E. N. Economou, and C. M. Soukoulis, Phys. Rev. B 83, 075108 (2011).

${ }^{14}$ Bo E. Sernelius, Phys. Rev. B 85, 195427 (2012).

${ }^{15}$ J. Sarabadani, A. Naji, R. Asgari, and R. Podgornik, Phys. Rev. B 84, 155407 (2011).

${ }^{16}$ D. Drosdoff and Lilia M. Woods, Phys. Rev. B 82, 155459 (2010).

${ }^{17}$ B. E. Sernelius, Europhys. Lett. 95, 57003 (2011).

${ }^{18}$ G. L. Klimchitskaya, U. Mohideen, and V. M. Mostepanenko, Int. J. Mod. Phys. B 25, 171 (2011).
${ }^{19}$ A. A. Banishev, C.-C. Chang, G. L. Klimchitskaya, V. M. Mostepanenko, and U. Mohideen, Phys. Rev. B 85, 195422 (2012).

${ }^{20}$ Norio Inui, J. Appl. Phys. 111, 074304 (2012).

${ }^{21}$ B. Geyer, G. L. Klimchitskaya, and V. M. Mostepanenko, Phys. Rev. B 81, 104101 (2010).

${ }^{22}$ D. Drosdoff and Lilia M. Woods, Phys. Rev. A 84, 062501 (2011).

${ }^{23}$ A. K. Geim, Science 324, 1530 (2009).

${ }^{24}$ M. Arif, K. Heo, B. Y. Lee, J. Lee, D. H. Seo, S. Seo, J. Jian, and S. Hong, Nanotechnology 22, 355709 (2011).

${ }^{25}$ Martin Pumera, Mater. Today 14, 308 (2011).

${ }^{26}$ Y. Shao, J. Wang, H. Wu, J. Liu, I. A. Aksay, and Y. Lin, Electroanalysis 14, 1027 (2010).

${ }^{27}$ F. Chen, Q. Qing, J. Xia, and N. Tao, Chem. Asian J. 5, 2144 (2010).

${ }^{28}$ Jin-Yong Hong and Jyongsik Jang, J. Mater. Chem. 22, 8179 (2012).

${ }^{29}$ M. Bordag, B. Geyer, G. L. Klimchitskaya, U. Mohideen, and V. M. Mostepanenko, Advances in the Casimir Effect (Oxford University Press, New York, 2009).

${ }^{30}$ B. Geyer, G. L. Klimchitskaya, and V. M. Mostepanenko, Ann. Phys. 323, 291 (2008).

${ }^{31}$ Daniel Garcia-Sanchez, King Yan Fong, Harish Bhaskaran, Steve Lamoreaux, and Hong X. Tang, Phys. Rev. Lett. 109, 027202 (2012).

${ }^{32}$ V. Svetovoy, Z. Moktadir, M. Elwenspoek, and H. Mizuta, Europhys. Lett. 96, 14006 (2011).

${ }^{33}$ D. Drosdoff, A. D. Phan, L. M. Woods, I. V. Bondarev, and J. F. Dobson, Eur. Phys. J. B (in press, 2012).

${ }^{34}$ George W. Hanson, IEEE Trans. Antennas Propag. 56, 747 (2008).

${ }^{35}$ Dimitrios L. Sounas and Christophe Caloz, IEEE Trans. Microwave Theory Tech. 60, 901 (2012). 